\title{
Usability of Destination-Sequenced Distance Vector Routing Protocol Routes
}

\author{
Miralem Mehic*§, Peppino Fazio $^{\S}$ and Miroslav Voznak ${ }^{\S}$ \\ * Department of Telecommunications, Faculty of Electrical Engineering, University of Sarajevo, \\ Zmaja od Bosne bb, Kampus Univerziteta, 71000 Sarajevo, Bosnia and Herzegovina \\ $\S$ VSB-Technical University of Ostrava, 17.listopadu 15, 70800 Ostrava-Poruba, Czech Republic
}

\begin{abstract}
The success of fundamental network tasks of traffic delivery from a source to a destination node is mainly dependent on the efficiency of the routing protocol. In mobile ad hoc networks, the effectiveness of routing protocols is additionally demanding due to the dynamic nature of network nodes. In this paper, we dealt with the exploitation of the routes generated using DSDV bellman-ford routing protocol. Through a total of 3960 network simulations with different topologies, network loads and mobility nodes, various parameters of the DSDV were considered. Our results show that there are a large number of unused routes, and techniques for improving the efficiency of routing and reducing routing overhead can be implemented.

Index Terms-routing protocols, networking, network performances, simulations
\end{abstract}

\section{INTRODUCTION}

The task of routing protocol is to analyze the state of the network and based on the information exchanged between neighbouring nodes, construct a view of network topology and find the optimal path ${ }^{1}$ between remote nodes (if such a path exists). The network topology reflects available destination nodes, and in general, depending on the search path timing, routing protocols can be divided into proactive, reactive, and hybrid. In the former, routes are searched only when needed, usually using flooding techniques [1], [2]. On the other hand, protocols that continuously exchange information about all possible paths are designated as proactive protocols. Such protocols, keep up-to-date information about the state of the network through exchanges of information on network topology or exchanges of routing tables even no data traffic exists [3], [4]. Aiming to simplify the problem by introducing a hierarchical network organization, hybrid protocols are based on the combination of proactive and reactive routing.

Unlike approaches dealing with the efficiency of routing protocols [5]-[7], defining optimal routing metrics [8]-[10] and analyzes of information propagation techniques [11], [12], in this paper, we deal with the practical utilization of routes defined by the proactive routing protocol. That is, we address the following questions: In a network where a proactive protocol is used, how many routes are actually used? More precisely, how much exchange of information on all the routes practically

This work was supported by the ESF in Science without borders project, reg. nr. CZ.02.2.69/0.0/0.0/16 027/0008463 within the Operational Programme Research, Development and Education

${ }^{1}$ In this paper, the paths and routes are used as synonyms, which denote the sequential series of nodes for connecting two distant nodes of the network. contributes to the utilization of the network? Our results show that a large number of routes in the network are not practically used, and techniques that optimize routing protocols (caching of routing entries, predictive routing, feedback routing and other) can be based on the obtained results to achieve higher routing optimization.

The rest of the paper is organized as follows: Section II provides the basis of proactive routing. The simulation setup is presented in section III while we provide an evaluation of the obtained result and discuss the broader aspects of our approach in section IV. Section V concludes this study and outlines future work.

\section{Proactive Routing Protocols}

This section provides basic information on the basics of proactive routing through the example of DSDV routing protocol. In the proactive routing approach, nodes are actively involved in route discovery and maintenance even when no data traffic exists. Therefore, routes are built, maintained and prepared for eventual data traffic, which generates more control/routing traffic in low-loaded networks. However, such an approach allows the delay to be reduced as the information on the established paths is ready and known in advance.

\section{A. Destination-Sequenced Distance-Vector Routing (DSDV)}

In this paper, DSDV is used to illustrate the proactive routing approach. We discuss DSDV, according to the definition of Perkins and Bhagwat (1994) [3]. DSDV is based on the distributed Bellman-Ford algorithm where each node maintains two tables of information. For each destination, $x$, each node $i$ keeps an address of node $k$ that can be used to reach destination $x$ as the next hop. DSDV requires each node to exchange and advertise its routing tables periodically. The traditional DSDV protocol settings are that periodic exchange of routing tables occurs every 15 seconds. Additionally, when receiving a DSDV packet, each node is required to analyze the values and further propagate them over the network to distribute the information to all nodes. There are different approaches to realize a consistent view of the network, and one of them is introducing a deliberate delay of smaller values that are added to 15 seconds to allow nodes to unite changes they receive from other nodes and then propagate merged values further into the network. The process is repeated on all nodes until all nodes receive propagated information. However, 
due to its distributed fashion, it is known that such kind of information exchange can lead to short-lived loops. Nodes due to the lack of a consistent view of the state of the network can route traffic using obsolete routing information, which can lead to traffic circulation. The simplest way to avoid the formation of routing loops is to mark routing entries with sequential numbers where a larger sequential number indicates fresher information. Next, each node uses triggered packets for fast propagation of values that can not wait for 15 seconds. However, if possible, the node will strive to merge triggered and periodic changes into one packet to minimize the information being sent. The period in which such an approach is considered is 5 seconds before sending a periodic report [4], [13]. It is important to note that DSDV uses the same message format for both periodicals and triggered reporting.

\section{Simulation Setup}

This section provides details about the setup of simulations that were used in our experiment.

To measure the amount, frequency and usability of defined routes, we simulated networks with random topologies consisting of 10, 20, 30, 40 and 50 nodes. We considered the impact of node mobility and the number of traffic-generating applications. The simulations were performed using the NS3 Simulator of version 3.28 [14]. For the same parameters of the number of nodes, the speed of movement, and the number of traffic-generating applications, 10 different scenarios were generated, which resulted in total 3960 simulations. The BRITE topology generator to generate random topologies since it is supported under NS-3 and the source code is freely available [15]. Table I lists the simulation parameters including parameters of $\mathrm{WiFi}$ NetDevices which were set to provide a maximal coverage area of $150 \mathrm{~m}^{2}$ and enable multihop communication. Parameters not given here are default parameters of the NS-3 v3.28 simulator.

TABLE I: Parameter values of the simulation

\begin{tabular}{|l|l|}
\hline Parameter & Value \\
\hline Simulation area & $1000 \times 1000 \mathrm{~m}^{2}$ \\
\hline Number of Nodes & $10,20,30,40,50$ \\
\hline Number of NetDevices per node & 1 \\
\hline Wifi Phy mode & DsssRate11Mbps \\
\hline Wifi Propagation Delay & Constant Speed Propagation Model \\
\hline Data Traffic Type & UDP Constant Bit Rate \\
\hline Data Traffic Rate & $512 \mathrm{kbps}$ \\
\hline Data Traffic Application & OnOffApplication \\
\hline Mobility Model & Random WayPoint \\
\hline Mobility Model Pause Interval & Constant $(1$ second $)$ \\
\hline Node speed in mobility model & $0,1,5,10,15,20,25,30,35,40,45,50 \mathrm{~m} / \mathrm{s}$ \\
\hline Total Simulation time & 250 seconds \\
\hline
\end{tabular}

The number of traffic-generated applications is defined depending on the total number of nodes $n$ in the following way:

- Simulations with a total of $1, n / 6, n / 5, n / 4, n / 3, n / 2$ and $n-1$ traffic applications are performed.

- For each of the applications, a node is randomly selected from the $(0,(n / 2)-1)$ range of nodes and the source traffic application is installed on the selected node.
- For each of the applications, a node is randomly selected from the $(n / 2, n-1)$ range of nodes and the destination traffic application is installed on the selected node.

Therefore, the network was loaded with traffic of constant intensity generated from randomly selected sources towards randomly selected destination nodes. In all simulations, UDP constant bit rate (CBR) OnOff applications with rate of 512 kbps were used.

To analyze the influence of mobility, we simulated networks using random waypoint mobility model with constant pause interval of 1 second and speeds of $0,1,5,10,15,2025$, $30,35,40,45$ and $50 \mathrm{~m} / \mathrm{s}$. Further, the DSDV routing tables are extended to keep information about the usage of routing entries. That is, each time the new entry to the routing table is added, the column "entriesUsed" is set to 0. The DSDV routing protocol is extended to increase the value of the field "entriesUsed" each time the routing entry is used. It is important to note, that local lookup actions ${ }^{2}$ are excluded from modifying the field since no routing actions are taken in such queries. Table II shows the example of extended DSDV routing table with "EntriesUsed" column included. No other changes to the simulator source code have been made in either the DSDV module or other modules. Thus, all simulations performed used the unchanged original source-code of the NS3 simulator with the modification described above that allowed the monitoring of the DSDV route usage.

\section{Simulation Results and Discussion}

This section provides basic results of the simulations conducted in our experiment.

\section{A. Paceket Delivery Ratio and Delay}

Fig. 1 shows the packet delivery ratio (the ratio of received and sent application packets) for different values of mobility. It shows that for the static network (mobility is $0 \mathrm{~m} / \mathrm{s}$ ) the PDR and the average delay (show in Fig. 2) values are maximal. However, with the increase of mobility, the values are reduced. For mobility values of $50 \mathrm{~m} / \mathrm{s}$, the PDR is slightly increased since the faster movement of nodes allows the faster and better utilization of network resources but with increased delay as it can be concluded from Fig. 2 .

\section{B. Forwarding Actions}

When the mobility of network nodes is increased, the routes are less stable, and multiple forwarding actions are needed to reach a final destination. Fig. 3 shows the impact of mobility on forwarding actions depicting that for values of 10 and 15 $\mathrm{m} / \mathrm{s}$ the maximal number of forwarding actions are recorded. When the network nodes move too fast, it is challenging to find suitable routes (as evident from PDR values) and forwarding actions are reduced as well.

\footnotetext{
${ }^{2}$ Local lookup actions include lookups whether a packet with DSDV entries carries new routing information or the information already exists in the local routing table.
} 
TABLE II: The example of DSDV routing table with the "EntriesUsed" column

\begin{tabular}{llllllll}
\hline Destination & Gateway & Interface & Hops & SeqNum & Lifetime & SettTime & EntriesUsed \\
\hline 10.1.1.1 & 10.1 .1 .1 & 10.1 .1 .3 & 1 & 4 & $0.018 \mathrm{~s}$ & $5.000 \mathrm{~s}$ & 6 \\
10.1 .1 .4 & 10.1 .1 .4 & 10.1 .1 .3 & 1 & 6 & $0.006 \mathrm{~s}$ & $5.000 \mathrm{~s}$ & 3 \\
10.1 .1 .255 & 10.1 .1 .255 & 10.1 .1 .3 & 0 & 6 & $0 \mathrm{~s}$ & $0 \mathrm{~s}$ & 61 \\
\hline
\end{tabular}
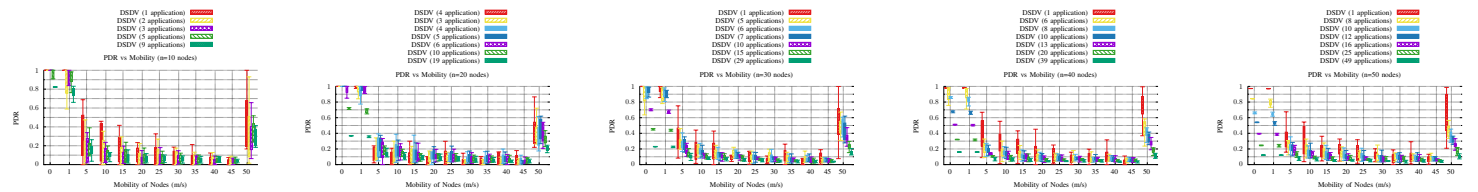

Fig. 1: Packet Delivery Ratio (the ratio of received and sent application packets) vs Mobility.
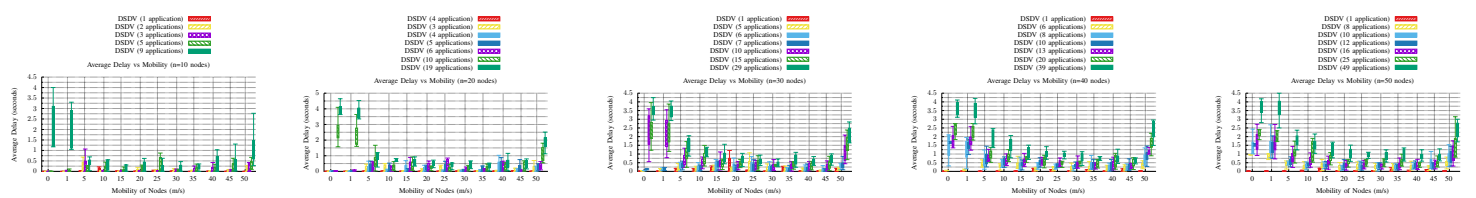

Fig. 2: Average delay of application packets vs Mobility.
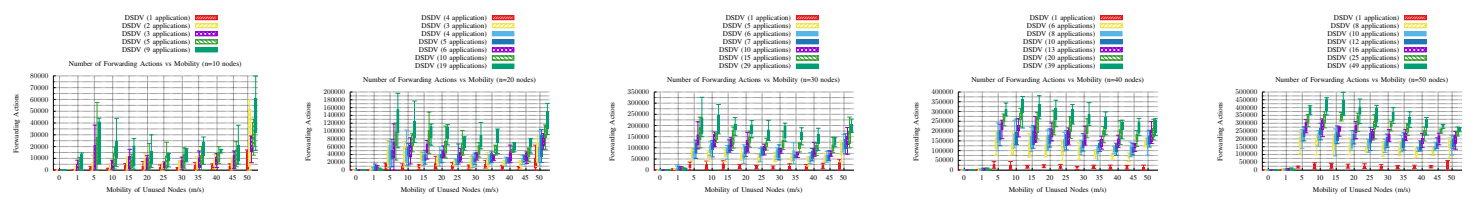

Fig. 3: Number of forwarding actions vs Mobility.
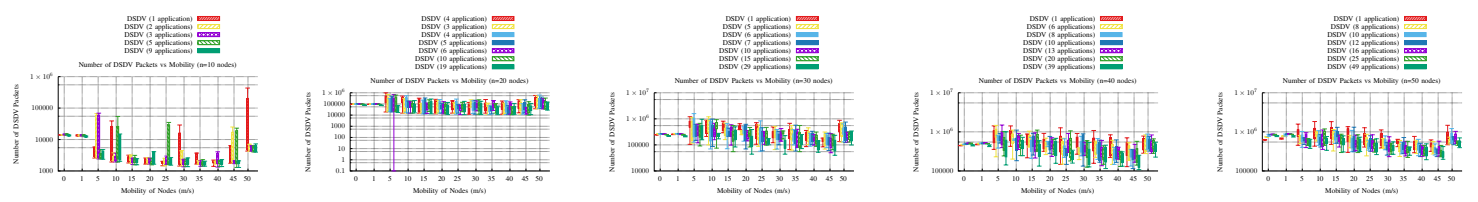

Fig. 4: Total number of exchanged DSDV routing packets vs Mobility.
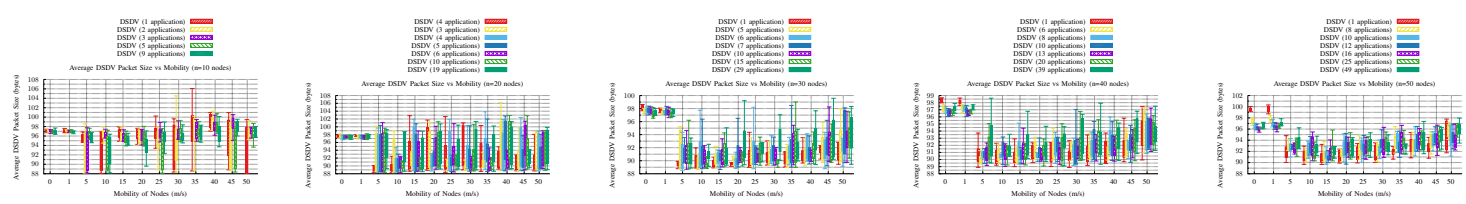

Fig. 5: Average size of DSDV routing packet vs Mobility.
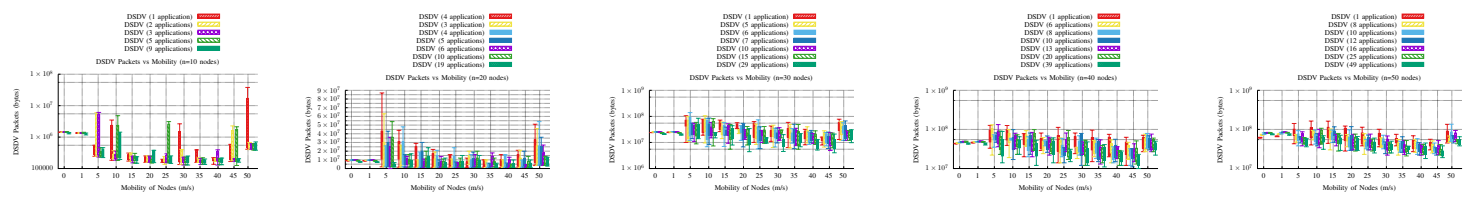

Fig. 6: Overall size of DSDV routing packet vs Mobility.
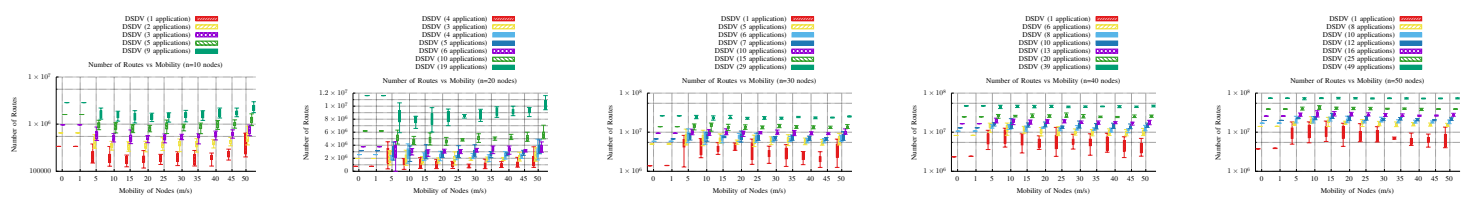

Fig. 7: Total number of DSDV routes vs Mobility. 

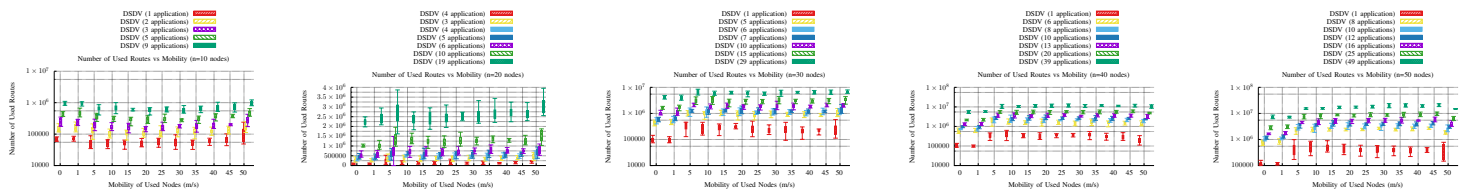

Fig. 8: Total number of used DSDV routes vs Mobility.
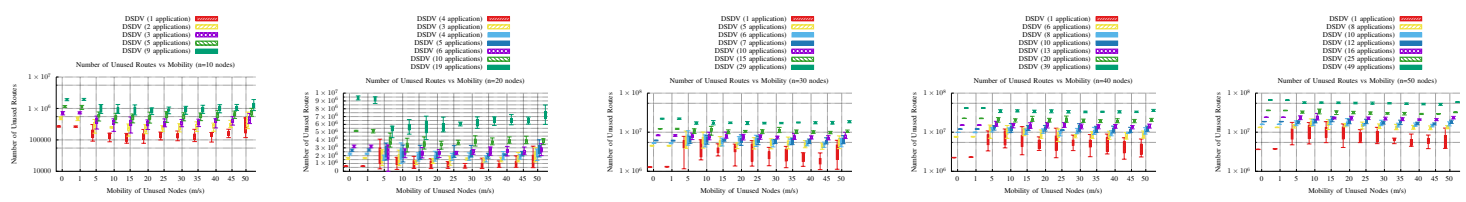

Fig. 9: Total number of unused DSDV routes vs Mobility.

\section{Routing Data}

As expected, for a network with more nodes, there are more routing packets to exchange, as shown in Fig. 4. However, the number of routing packets decreases when mobility increases. For static networks (mobility $0 \mathrm{~m} / \mathrm{s}$ ) the number of exchanged routing packets is fixed regardless of traffic load. However, the number of triggered DSDV packets (packets not waiting for 15 seconds of periodic route update interval) is increasing when mobility increases. As shown in Fig. 5 and Fig. 6, the size of routing packets varies for different mobility but in general the advertise packets are decreasing for increased mobility (Fig. 6).

\section{Route usage}

Our results show that in average $19.428 \%$ of generated and exchanged routing tables are used ${ }^{3}$. In networks with 10 nodes the average usage of routing entries is $32.788 \%$, in networks with 20 nodes the average usage is $20.505 \%$, in networks with 30 nodes the average usage is $16.847 \%$, in networks with 40 nodes the average usage is $15.897 \%$ while in networks with 50 nodes the average usage is $14.942 \%$. Fig. 7 shows the overall number of established routes, Fig. 8 shows the number of used routes while Fig. 9 shows the overall number of unused routes.

It is worth considering the impact of network load. When the network is loaded with only one application generating the traffic, the average route usage was $26.52 \%$ for $\mathrm{n}=10$ nodes; $13.62 \%$ for $\mathrm{n}=20$ nodes; $8.01 \%$ for $\mathrm{n}=30$ nodes; $5.842 \%$ for $\mathrm{n}=40$ nodes and $4.806 \%$ for $\mathrm{n}=50$ nodes. When the network is loaded with additinal traffic in the way that each node is either source or destination of application traffic, the average route usage was $38.4 \%$ for $n=10$ nodes; $28.163 \%$ for $n=20$ nodes; $24.595 \%$ for $\mathrm{n}=30$ nodes; $22.692 \%$ for $\mathrm{n}=40$ nodes and $22.137 \%$ for $\mathrm{n}=50$ nodes.

\section{CONCLUSION}

This paper addressed the question of the usability of destination-sequenced distance vector routing protocol routes. The work summarized the measurements that were performed

\footnotetext{
${ }^{3}$ Here, we explicitly refer to the records that exist in routing tables and were used for routing.
}

TABLE III: Minimum, maximum, and average route usage statistics for different network loads and networks of 10, 20, 30,40 , and 50 nodes.

\begin{tabular}{|c|c|c|c|c|}
\hline & & \multicolumn{3}{|c|}{ Route Usage (\%) } \\
\hline Nodes & Applications & Min & Max & Average \\
\hline 10 & 1 & 0.119 & 0.468 & 0.265 \\
\hline 10 & 2 & 0.179 & 0.516 & 0.298 \\
\hline 10 & 3 & 0.181 & 0.572 & 0.328 \\
\hline 10 & 5 & 0.219 & 0.530 & 0.353 \\
\hline 10 & 9 & 0.271 & 0.556 & 0.394 \\
\hline 20 & 1 & 0.064 & 0.341 & 0.136 \\
\hline 20 & 3 & 0.09 & 0.303 & 0.181 \\
\hline 20 & 4 & 0.09 & 0.288 & 0.183 \\
\hline 20 & 5 & 0.09 & 0.321 & 0.196 \\
\hline 20 & 6 & 0.11 & 0.349 & 0.214 \\
\hline 20 & 10 & 0.123 & 0.343 & 0.243 \\
\hline 20 & 19 & 0.170 & 0.416 & 0.281 \\
\hline 30 & 1 & 0.039 & 0.161 & 0.08 \\
\hline 30 & 5 & 0.067 & 0.219 & 0.141 \\
\hline 30 & 6 & 0.067 & 0.259 & 0.154 \\
\hline 30 & 7 & 0.07 & 0.239 & 0.158 \\
\hline 30 & 10 & 0.089 & 0.304 & 0.189 \\
\hline 30 & 15 & 0.090 & 0.316 & 0.209 \\
\hline 30 & 29 & 0.121 & 0.327 & 0.245 \\
\hline 40 & 1 & 0.033 & 0.106 & 0.058 \\
\hline 40 & 6 & 0.053 & 0.291 & 0.140 \\
\hline 40 & 8 & 0.054 & 0.258 & 0.151 \\
\hline 40 & 10 & 0.054 & 0.247 & 0.163 \\
\hline 40 & 13 & 0.067 & 0.275 & 0.177 \\
\hline 40 & 20 & 0.067 & 0.285 & 0.194 \\
\hline 40 & 39 & 0.097 & 0.338 & 0.226 \\
\hline 50 & 1 & 0.025 & 0.094 & 0.048 \\
\hline 50 & 8 & 0.041 & 0.205 & 0.127 \\
\hline 50 & 10 & 0.052 & 0.229 & 0.142 \\
\hline 50 & 12 & 0.051 & 0.243 & 0.152 \\
\hline 50 & 16 & 0.053 & 0.259 & 0.164 \\
\hline 50 & 25 & 0.060 & 0.289 & 0.190 \\
\hline 50 & 49 & 0.084 & 0.327 & 0.221 \\
\hline
\end{tabular}

using NS-3 v3.28 ${ }^{4}$ simulator with BRITE random topology generation. Our measurements of 3960 simulations, showed that, in general, $19.32 \%$ routes were used. In the best case,

\footnotetext{
${ }^{4}$ The latest version of the simulator that was available at the time of experimenting. However, no significant changes to the modules: DSDV, mobility were not made in version 3.29 and 3.30 (https://www.nsnam.org/news/2019/08/21/ns-3-30-released.html), and we are convinced that identical results can be achieved using the versions mentioned above of the NS-3 simulator.
} 
the usage of DSDV routes with the value of $43.07 \%$ was recorded in the network with ten nodes where each node was used as either a source or destination of application data. That is, the rest of the exchanged routes were not used. The obtained results show there is an open space for improving the efficiency of routing and reducing routing overhead data which is important from various aspects (such as energy consumption in sensor and wireless networks and other).

The main contribution of this paper is the analysis of the performance of DSDV routing protocol for different settings mobile ad-hoc networks considering mobility, a number of nodes and network load. Our future work will focus on techniques and approaches for improving the reduction of routing overhead and increasing the practical usability of routing entries. Also, we plan to gain knowledge and comparable results when investigating different routing protocols.

\section{REFERENCES}

[1] C. Perkins et al., "Ad-hoc On-Demand Distance Vector Routing," 2003.

[2] P. Fazio et al., "An on demand interference aware routing protocol for VANETS," Journal of Networks, vol. 7, pp. 1728-1738, 2012.

[3] C. E. Perkins et al., "Highly dynamic Destination-Sequenced DistanceVector Routing (DSDV) for Mobile Computers," ACM SIGCOMM Computer Communication Review, vol. 24, no. 4, pp. 234-244, 1994.

[4] M. Mehic et al., "On using multiple routing metrics with destination sequenced distance vector protocol for MultiHop wireless ad hoc networks," S. Harkrider et al., Eds. International Society for Optics and Photonics, may 2016, p. 98480F.

[5] F. De Rango et al., "Link-Stability and Energy Aware Routing Protocol in Distributed Wireless Networks," IEEE Transactions on Parallel and Distributed Systems, vol. 23, no. 4, pp. 713-726, 2012.

[6] F. Li et al., "Routing in vehicular ad hoc networks: A survey," IEEE Vehicular Technology Magazine, vol. 2, no. 2, pp. 12-22, 2007.

[7] M. V. Khiavi et al., "Performance Comparison of AODV , DSDV , DSR and TORA Routing Protocols in MANETs," International Research Journal of Applied and Basic Sciences, vol. 3, no. 7, pp. 1429-1436, 2012.

[8] Y. Yang et al., "Designing Routing Metrics for Mesh Networks," IEEE Workshop on Wireless Mesh Networks, 2005.

[9] _ - "Design Guidelines for Routing Metrics in Multihop Wireless Networks," in 2008 Proceedings IEEE INFOCOM - The 27th Conference on Computer Communications. IEEE, apr 2008, pp. $1615-1623$.

[10] N. Javaid et al., "Identifying Design Requirements for Wireless Routing Link Metrics," GLOBECOM - IEEE Global Telecommunications Conference, pp. 1-5, 2011.

[11] M. Kassim et al., "Mobile ad hoc network (MANET) routing protocols comparison for wireless sensor network," 2011 IEEE International Conference on System Engineering and Technology, pp. 148-152, 2011.

[12] A. Boukerche, Algorithms and Protocols for Wireless Sensor Networks, 2009 , vol. 6

[13] S. R. Das et al., "Simulationbased performance evaluation of routing protocols for mobile ad hoc networks," Mobile Networks and Applications, vol. 5, no. 3, pp. 179-189, 2000.

[14] T. R. Henderson et al., "Network Simulations with the ns-3 Simulator," Proc. Sigcomm, p. 527, 2006.

[15] A. Medina et al., "BRITE: an approach to universal topology generation," in MASCOTS 2001, Proceedings Ninth International Symposium on Modeling, Analysis and Simulation of Computer and Telecommunication Systems. IEEE Comput. Soc, 2001, pp. 346-353. 